\title{
Synthesis of vibration waves based on wavelet technology
}

\author{
L.H. Zou ${ }^{\mathrm{a}}$, A.P. Liu ${ }^{\mathrm{b}}$, X. Mac,*, C. Zhang ${ }^{\mathrm{d}}$ and K. Huang ${ }^{\mathrm{a}}$ \\ ${ }^{a}$ Department of Civil Engineering, Fuzhou University, Fuzhou, China \\ ${ }^{\mathrm{b}}$ Department of Civil Engineering, Lanzhou Jiaotong University, Lanzhou, China \\ ${ }^{\mathrm{c}}$ School of Natural and Built Environments, University of South Australia, Adelaide, Australia \\ ${ }^{\mathrm{d}}$ Department of civil and Environmental Engineering, University of Auckland Auckland, New Zealand
}

Received 8 February 2010

Revised 28 August 2010

\begin{abstract}
A novel method to generate time series of vibration waves is proposed in the paper. Considering the frequency band energy as the criterion, synthesis formulas for fluctuating wind pressure and earthquake ground motion are developed in terms of Daubechies wavelet and Harr wavelet respectively. The wavelet reconstruction method is applicable to both stationary and non-stationary process simulation. Theoretically, for non-stationary (such as seismic) process synthesis, it has a better non-stationarity in time-frequency domain than the traditional trigonometric series. Influence of wavelet delamination number and wavelet function type is also analyzed. Numerical results show that the synthesis of vibration waves based on wavelet reconstruction method contains main components of vibration, and can reflect the main properties of practical vibrations.
\end{abstract}

Keywords: Vibration, wavelet, non-stationary, synthesis, wind load, seismic excitation

\section{Introduction}

Due to the lack of time-history recorders, artificial wave samples are commonly used to simulate the fluctuation in wind loads and seismic excitations during structural dynamic analysis.

The idea of random wave synthesis appeared in 1940s. Early studies were focused on simulation of white-noise ground motions through one-cycle sine-wave pulses [6,8]. Although these artificial time functions were based on the stationary single-dimensional, single-variate Gaussian processes stochastic process, the pioneering works made it possible to study linear and nonlinear seismic effect on the basis of probability methods. The method was then improved by Shinozuka [17,18] to simulate multidimensional, multivariate non-Gaussian cases through using cosine series. Because the trigonometric method was a good tool for processing stationary signals in time domain and/or frequency domain, it was later widely and successfully applied in wind engineering field to generate artificial Gaussian or Non-Gaussian fluctuating wind velocity samples $[2,10,13]$.

For seismic wave synthesis, recorded accelerograms show clear non-stationary of strong ground motion which can influence structural responses significantly. To meet the non-stationary frequency and amplitude characteristics, stationary random accelerograms were multiplied by a non-stationary envelope function with the frequency contents and phases of the trigonometric time series changed to a time-dependent function $[3,5,7,11,12,16,19,21]$. Thus the ensemble averages of the modified accelerograms would not keep constant in time-domain and might be considered as "non-stationary". However, the statistical characteristics of the accelegrom samples derived from the time-dependent amplitude enveloping functions and frequency functions, are still time-dependent, but not of the real meaning of

\footnotetext{
*Corresponding author. E-mail: xing.ma@unisa.edu.au.
} 
non-stationarity. Theoretically, the artificial seismic wave synthesized by trigonometric series method is not able to describe local non-stationary properties of time-frequency. Fortunately, wavelet analysis [4,14], a method of timefrequency analysis, can overcome the shortages of Fourier analysis. It has a variable time-frequency window, which can widen (or narrow) automatically for high (or low) signal components and can be easily applied for generation of non-stationary signals. The purpose of this paper is to synthesize the stationarity and non-stationarity vibration waves using the wavelet reconstruction method, and to study its characteristics.

\section{Synthesis of vibration waves based on trigonometric series method}

\subsection{Fluctuating wind (stationarity process)}

The fluctuating wind is generally regarded as a stationarity process [20]. The idea of synthesis for fluctuating wind waves based on trigonometric series is to construct a gauss random stationarity process and obtain the sum of the trigonometric series. According to Shinozuka [17,18], the random sampling of fluctuating wind can be obtained as:

$$
g(t)=\sum_{i=1}^{N} \sqrt{2 S_{V}(\omega) \Delta \omega} \cos \left(\omega_{i} t+\phi_{i}\right)
$$

Where, $S_{V}(\omega)$ is the power spectral density function of random sample $g(t) ; \Delta \omega$ is the increment of frequency; $\phi_{i}$ is the phase angle of $\mathrm{i}$-th frequency component, which is uniformly distributed in the scope of $[0,2 \pi] ; \omega_{i}$ is the $\mathrm{i}$-th frequency; $N$ is a large positive integer. According to Davenport, the spectral function for fluctuating wind speed can be expressed as:

$$
\left\{\begin{array}{c}
S_{V}(f)=4 K \bar{v}_{10}^{2} \frac{x^{2}}{f\left(1+x^{2}\right)^{4 / 3}} \\
x=\frac{1200 f}{\bar{v}_{10}}
\end{array}\right.
$$

where, $K$ is the surface drag coefficient; $\bar{v}_{10}$ is the average wind speed at the height of $10 \mathrm{~m}$ above the ground.

\subsection{Seismic (non-stationarity process)}

Similar to the fluctuating wind, the idea of synthetic seismic waves is also to construct a gauss random stationary process with the sum of a group of the trigonometric series [1]. The popular model is:

$$
a(t)=f(t) \sum_{k=n_{1}}^{n_{2}} c_{k} \cos \left(\omega_{k}+\varphi_{k}\right)
$$

where, $\omega_{k}=2 k \pi / T, \omega_{k}$ is the k-th frequency component; $n_{1}$ and $n_{2}$ defines the scope of frequency summing; $\varphi_{k}$ is the phase angle of the k-th frequency component, which is uniformly distributed in the scope of $[0,2 \pi]$; and $c_{k}$ is amplitude of the k-th harmonic component, which can be arbitrarily given or determined by the response spectrum and the power spectrum.

$$
c_{k}=2 \sqrt{\frac{-\zeta}{k \pi|\ln A|}} S_{T}\left(2 \pi / \omega_{k}\right)
$$

where $A=[-\ln (1-P)-\ln (2 \pi)]$, and $S_{T}\left(2 \pi / \omega_{k}\right)$ is the value of target spectrum in $2 \pi / \omega_{k} ; P$ is the probability that the power spectrum exceeds the target value $S_{T}\left(2 \pi / \omega_{k}\right)$ at the period of $2 \pi / \omega_{k}$, and $\zeta$ is the damping ratio.

Envelope function $f(t)$ generally use the segment function given as follows [9,15] (Fig.1):

$$
f(t)=\left\{\begin{array}{cc}
t^{2} / t_{1}^{2} & 0 \leqslant t<t_{1} \\
1 & t_{1} \leqslant t<t_{2} \\
e^{-\alpha\left(t-t_{2}\right)} & t_{2} \leqslant t
\end{array}\right.
$$

$\alpha$ is the attenuation index when $t \geqslant t_{2}$ 


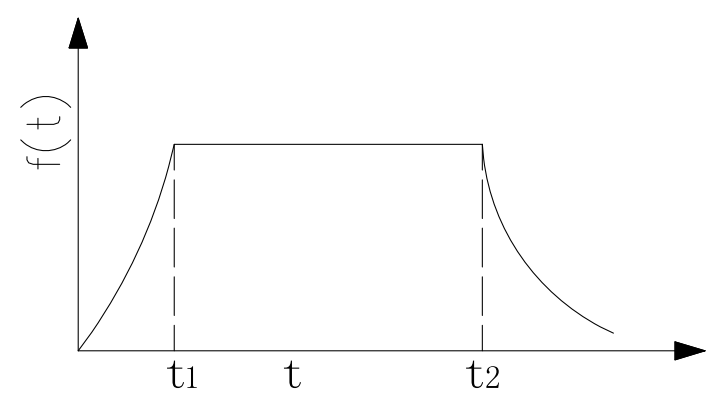

Fig. 1. Envelop function $f(\mathrm{t})$.

\section{Wavelet reconstruction theory}

If $x(t)$ is a square integrable function [ hereinafter as $\left.x(t) \in L^{2}(R)\right], \psi(t)$ is defined as the function of basic wavelet or mother wavelet [4], then:

$$
W T_{x}(\alpha, \tau)=\frac{1}{\sqrt{a}} \int x(t) \psi^{*}\left(\frac{t-\tau}{a}\right) d t=\left\langle\left(x(t), \psi_{a \tau}(t)\right\rangle\right.
$$

Where, $a$ is the size factor with $a>0 ; \tau$ is the translation parameter; $\psi^{*}(t)$ is the complex conjugate of

$$
\psi(t) ; \psi_{a \tau}(t)=\frac{1}{\sqrt{a}} \psi\left(\frac{t-\tau}{a}\right)
$$

is the displacement and size expansion function of mother wavelet.

When

$$
c_{\psi}=\int_{-\infty}^{\infty} \frac{|\Psi(\omega)|^{2}}{\omega} d \omega<\infty
$$

$x(t)$ can be reconstructed by $W T_{x}(a, \tau)$ as [4]:

$$
\begin{aligned}
x(t) & =\frac{1}{c_{\psi}} \int_{0}^{\infty} \frac{d a}{a^{2}} \int_{-\infty}^{\infty} W T_{x}(a, \tau) \psi_{a \tau}(t) d \tau \\
& =\frac{1}{c_{\psi}} \int_{0}^{\infty} \frac{d a}{a^{2}} \int_{-\infty}^{\infty} W T_{x}(a, \tau) \frac{1}{\sqrt{a}} \psi\left(\frac{t-\tau}{a}\right) d \tau
\end{aligned}
$$

$c_{\psi}=\int_{-\infty}^{\infty} \frac{|\Psi(\omega)|^{2}}{\omega} d \omega<\infty$ is the admissible condition.

\section{Synthesis of vibration waves based on wavelet method}

\subsection{Fluctuating wind}

According to quasi-linear response spectrum, using wavelet function to replace harmonic wave and wavelet coefficient $W T_{g}(a, \tau)$ to replace amplitude $\sqrt{2 S_{V}(\omega) \Delta \omega}$ in Eq. (1), fluctuating wind wave can be synthesized.

A lemma similar to Basel's theorem can be derived from Moyal formula, showing that the integral of the square of wavelet coefficient is proportional to signal energy [4].

$$
\int_{0}^{\infty} \frac{d a}{a^{2}} \int_{-\infty}^{\infty}\left|W T_{g}(a, \tau)\right|^{2} d \tau=c_{\psi} \int_{-\infty}^{\infty}|g(t)|^{2} d t
$$

If $g(t)$ is fluctuating wind signal, its total energy is:

$$
E=\frac{1}{c_{\psi}} \int_{0}^{\infty} \frac{d a}{a^{2}} \int_{-\infty}^{\infty}\left|W T_{g}(a, \tau)\right|^{2} d \tau
$$


By representing the scale factor $a$ and the translation factor $\tau$ in dyadic discrete, Eq. (9) can be written as:

$$
E=\frac{3}{4 c_{\psi}} \sum_{j} \sum_{k} W T_{g}(j, k)^{2}
$$

Wavelet's energy function at $j$ can be obtained as:

$$
\left\{\begin{array}{c}
E=\sum_{j} E_{j} \\
E_{j}=\frac{3}{4 c_{\psi}} \sum_{k} W T_{g}(j, k)^{2}
\end{array}\right.
$$

where $E_{j}$ is the total wavelet energy at $j$ and also the total energy of signal in frequency band at $j ; j$ is the resolution of wavelet

$$
E_{j}=\int_{[\omega]} T S(\omega) d \omega
$$

T is the duration time of signal; $S(\omega)$ is the power spectrum. When $E_{j}$ is known, wavelet coefficient can be obtained as

$$
W T_{g}(j, k)=\sqrt{\frac{4 c_{\psi}}{3}} S^{\prime}(j, k) \sqrt{\frac{E_{j}}{S^{\prime}(j)}}
$$

where $S^{\prime}(j, k)$ is the random number when the group at $j$ is a uniform distribution on $[-1,1]$; the amount of random number in $j$ group $k=T \cdot 2^{j}$ ( $T$ is the duration time); $S^{\prime}(j)$ is the quadratic sum. According to the energy conservation principle of frequency band and Eq. (1), the fluctuating wind can be obtained:

$$
g(t)=\frac{3}{4 c_{\psi}} \sum_{j} \sum_{k} W T_{g}(j, k) \psi_{j, k}(t)
$$

By solving the fluctuating wind time-history $g(t)$, and reversely calculating the energy values $E_{j}^{i}$ in every spectrum (superior figures $i$ represent iteration number), the error can be estimated by:

$$
\left(E_{j}^{i}-E_{j}\right) / E_{j} \leqslant K
$$

where $K$ is the maximum allowable error; $E_{j}$ is the sub-frequency band energy calculated from designed response spectrum; $E_{j}^{i}$ is the sub-frequency energy, which is calculated from fluctuating wind produced by i-th iteration. The wavelet coefficient should be modulated if the Eq. (15) is not satisfied:

$$
W T_{g}^{i+1}(j, k)=W T_{g}^{i}(j, k) \cdot \sqrt{E_{j} / E_{j}^{i}}
$$

\subsection{Seismic}

Similar to fluctuating wind, to synthesis seismic wave based on wavelet reconstruction method, harmonic wave function need to be replace by the wavelet function. Accordingly, amplitude $c_{k}$ in Eq. (3) need to be replaced by wavelet coefficient $W T_{x}(a, \tau)$ and seismic wave can be synthesized according to response spectrum quasi-linear theroy.

$$
\int_{0}^{\infty} \frac{d a}{a^{2}} \int_{-\infty}^{\infty}\left|W T_{a}(a, \tau)\right|^{2} d \tau=c_{\psi} \int_{-\infty}^{\infty}|a(t)|^{2} d t
$$

Total energy of the seismic signal is:

$$
E=\frac{1}{c_{\psi}} \int_{0}^{\infty} \frac{d a}{a^{2}} \int_{-\infty}^{\infty}\left|W T_{a}(a, \tau)\right|^{2} d \tau
$$

Similar to fluctuating wind, the seismic acceleration can be obtained after a series of transforms: 


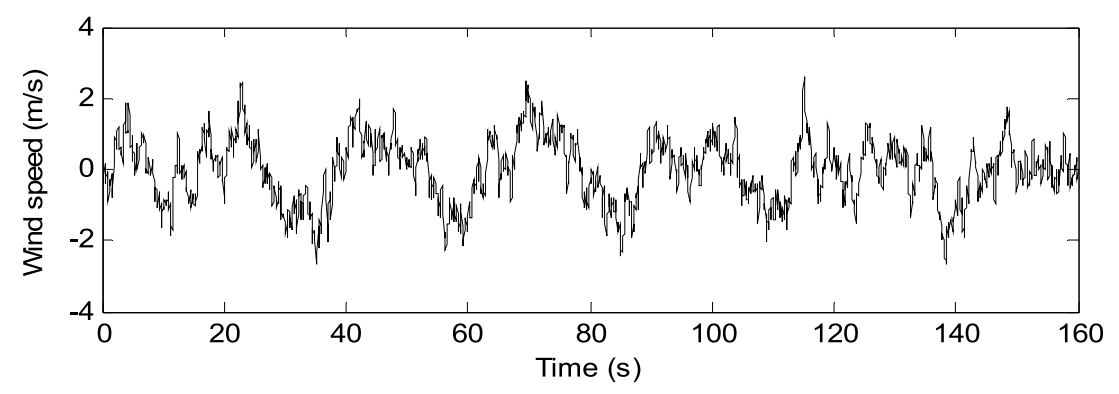

Fig. 2. Speed time history of fluctuating wind.

$$
\left.a(t)=\frac{3}{4 c_{\psi}} f_{(} t\right) \sum_{j} \sum_{k} W T_{a}(j, k) \psi_{j, k}(t)
$$

In Eq. (12), the relationship between power spectrum and response spectrum is:

$$
S(\omega)=\frac{2 \zeta}{\pi \omega} S_{a}(\omega)^{2} /\left\{-2 \ln \left[-\frac{\pi}{\omega T} \ln (1-P)\right]\right\}
$$

where $S_{a}$ is the designed response spectrum. $[\omega]$ is frequency band at $j$.

Unlike fluctuating wind, the seismic is a classical non-stationary process; consequently, an envelope function $f(t)$ should be multiplied in Eq. (19).

By solving the acceleration response time-history $a(t)$, calculating energy values $E_{j}^{i}$ in every spectrum based on $a(t)$, the error can be estimated by:

$$
\left(E_{j}^{i}-E_{j}\right) / E_{j} \leqslant K
$$

The wavelet coefficient should be modulated if the Eq. (21) is not satisfied:

$$
W T_{a}^{i+1}(j, k)=W T_{a}^{i}(j, k) \cdot \sqrt{E_{j} / E_{j}^{i}}
$$

\section{Numerical simulation}

\subsection{Fluctuating wind}

In Eq. (1), $S_{V}(f)$ is the speed spectrum of fluctuating wind. Actually, the pressure spectrum is more common to be used in practical application. According to the definition of pressure spectrum for fluctuating wind and the theorem of Wiener-Khintchine, the power spectrum of fluctuating wind can be obtained as:

$$
S_{W}(f)=\rho^{2} \bar{v}_{z}^{2} S_{V}(f)=16 K \bar{\omega}_{z}^{2} \frac{\bar{v}_{10}^{2}}{\bar{v}_{z}^{2}} \frac{x^{2}}{f\left(1+x^{2}\right)^{4 / 3}}
$$

where $S_{W}(f)$ is the power spectrum function of fluctuating wind; $\rho$ is the density of atmosphere; $\bar{v}_{z}$ is the wind speed at the height of $z ; \bar{\omega}_{z}$ is the wind pressure at the height of $z$. Thus, using the pressure power spectrum of fluctuating wind, the time-history of pressure can be simulated.

Example 1 Determine the time-history of fluctuating wind waves for some site-based wavelet.

Conditions: heigh: $40 \mathrm{~m}$; duration time: $160 \mathrm{~s}$; the surface drag coefficient $K=0.0022$; the density of atmosphere $\rho=1 / 820$; the basic speed of wind $\bar{v}_{10}=23.9 \mathrm{~m} / \mathrm{s}$; the number of samples $\mathrm{N}=2048$; the time step: $0.0781 \mathrm{~s}$.

The time histories of fluctuating wind speed and pressure based on the wavelet of Daubechies (db3) are simulated in Figs 2 and 3, and the power spectrums of fluctuating wind speed and pressure are shown in Figs 4 and 5 accordingly.

As shown in Figs 4 and 5, almost all the energy of fluctuating wind distributes within the range of $0 \sim 3 \mathrm{~Hz}$, and is mostly concentrated in the range of $0 \sim 1 \mathrm{~Hz}$ with the largest power spectrum value located around $0.1 \mathrm{~Hz}$. As 


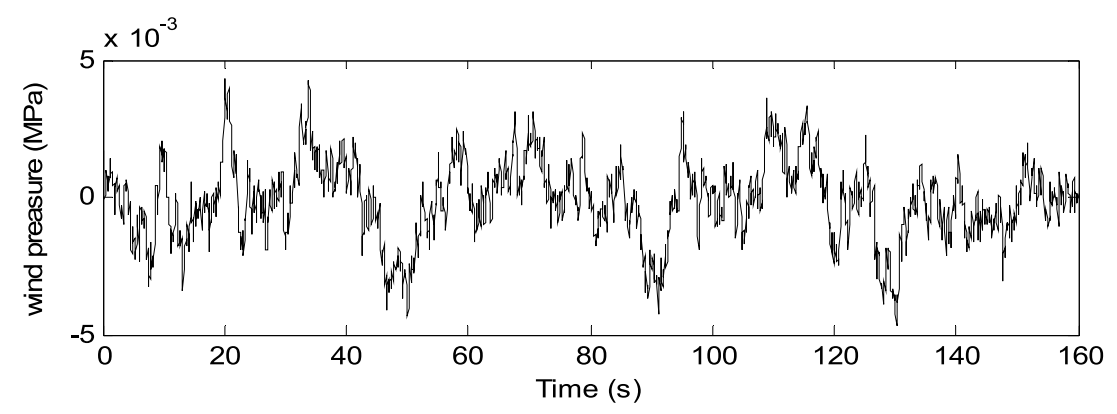

Fig. 3. Pressure time history of fluctuating wind.

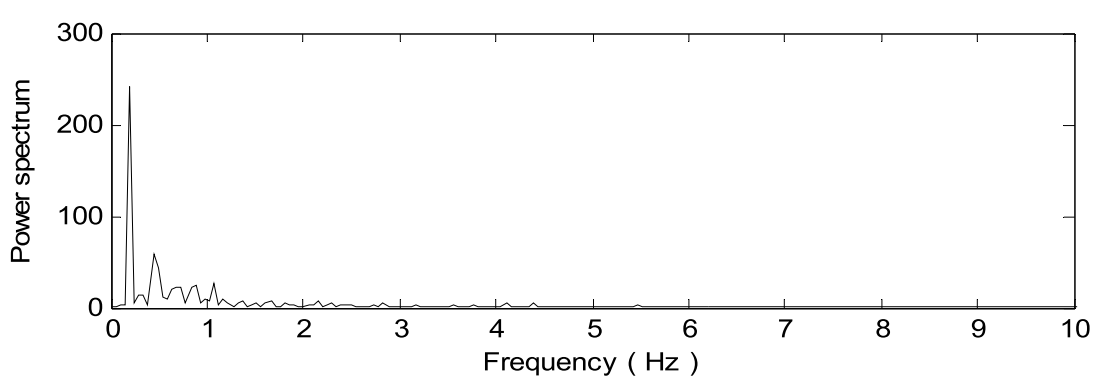

Fig. 4. Speed power spectrum of fluctuating wind.

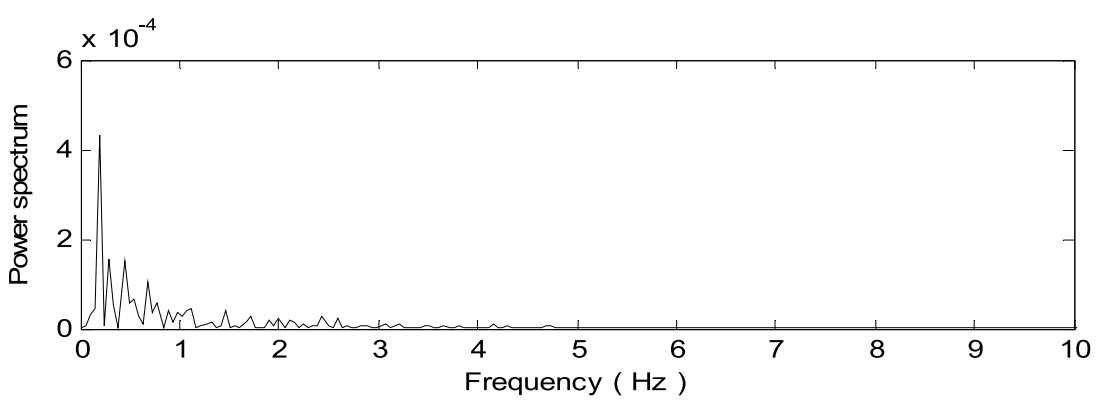

Fig. 5. Pressure power spectrum of fluctuating wind.

a result, high and pliant structures with long periods, are especially sensitive to fluctuating winds. Therefore, wind responses are crucial design factors for these long period structures.

According to the sampling theorem, when the frequency of sampling is greater than twice of the maximum frequency of signal, there will be no distortion. In Figs 4 and 5, almost all of the energy concentrates within the frequency band of $0 \sim 5 \mathrm{~Hz}$, therefore, the sampling frequency of $10 \mathrm{~Hz}$ is appropriate.

Because the speed spectrum of Davenport fluctuating wind doesn't change with the variation of space, the fluctuating wind speed at different coordinates (height and horizontal location) should be obtained through the relationship between the auto power spectrum and the cross power spectrum.

\subsection{Seismic}

The seismic acceleration design response spectrum curve in Fig. 6 is from anti-seismic design code of China.

In Fig. 6, $\alpha$ is the seismic effect coefficient; $\alpha_{\max }$ is the maximum of seismic effect coefficient; $\eta_{1}$ is the adjustment coefficient; $\gamma$ is attenuation index; $T_{g}$ is the characteristic period; $\eta_{2}$ is damping adjustment coefficient; $T$ is the structural natural period. 
Table 1

The overburden thickness of various site types $/ \mathrm{m}$

\begin{tabular}{lcccc}
\hline Shear-wave & \multicolumn{5}{c}{ Site types } \\
\cline { 2 - 5 } velocity/m/s & I & II & III & IV \\
\hline$v_{s e}>500$ & 0 & & & \\
$500 \geqslant v_{s e}>250$ & $<5$ & $\geqslant 5$ & & \\
$250 \geqslant v_{s e}>140$ & $<3$ & $3 \sim 50$ & $>50$ & \\
$v_{s e} \leqslant 140$ & $<3$ & $3 \sim 15$ & $>15 \sim 80$ & $>80$ \\
\hline
\end{tabular}

Table 2

Maximum value of horizontal seismic influencing coefficient

\begin{tabular}{lcccc}
\hline Seismic effect & 6 degree & 7 degree & \multicolumn{1}{c}{8 degree } & 9 degree \\
\hline Small seismic & 0.04 & $0.08(0.12)$ & $0.16(0.24)$ & 0.32 \\
Strong seismic & - & $0.50(0.72)$ & $0.90(1.2)$ & 1.4 \\
\hline
\end{tabular}

Note: For cases with the design seismic accelerations of $1.5 \mathrm{~m} / \mathrm{s}^{2}(7$ degree) or $3 \mathrm{~m} / \mathrm{s}^{2}$ ( 8 degree), values in the brackets are applied.

Table 3

Characteristic period value (s)

\begin{tabular}{lllll}
\hline Classification of & \multicolumn{4}{c}{ Site subsoil categories } \\
\cline { 2 - 5 } seismic design & I & II & III & IV \\
\hline First group & 0.25 & 0.35 & 0.45 & 0.65 \\
Second group & 0.3 & 0.4 & 0.55 & 0.75 \\
Third group & 0.35 & 0.45 & 0.65 & 0.9 \\
\hline
\end{tabular}

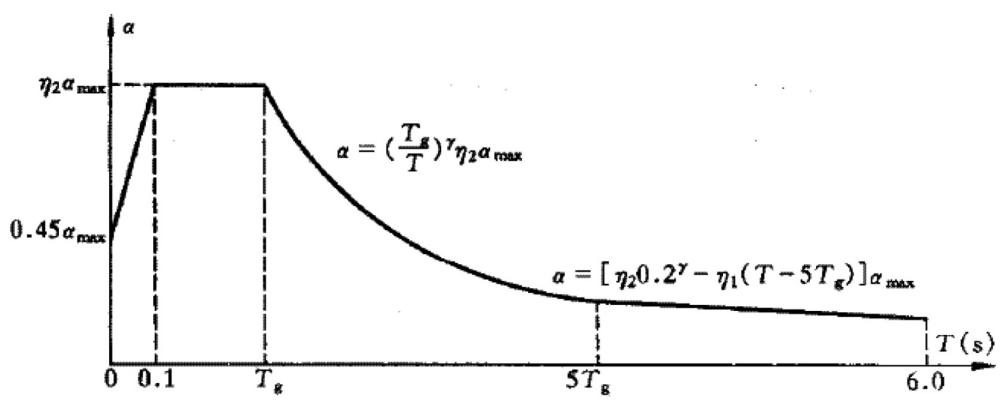

Fig. 6. Curve of acceleration response spectrum.

$$
\left\{\begin{array}{l}
\gamma=0.9+\frac{0.05-\zeta}{0.5+5 \zeta} \\
\eta_{1}=0.02+(0.05-\zeta) / 8 \\
\eta_{2}=1+\frac{0.05-\zeta}{0.06+1.7 \zeta}
\end{array}\right.
$$

where $\eta_{2}=0.55$ if $\eta_{2}<0.55$ based on calculation.

In the anti-seismic design code of China, the site is divided into four types according to the equivalent shear-wave velocity of soil and the thickness of overburden.

Example 2. In simulation, Daubechies $(\mathrm{db} 3)$ is chosen as the wavelet function. Consider an earthquake with the following parameters: the duration time $=16 \mathrm{~s}$; the exceeding probability $=63 \%$; the damping ratio $\zeta=0.05$; seismic design group $=3$. The maximum values of horizontal seismic influencing coefficient are shown in Table 2 . The characteristic period values in terms of site subsoil categories are shown in Table 3. Based on numerical simulation, the seismic accelerations and corresponding power spectrum curves for type II, III, IV sites in 8 degree areas are synthesized (Figs 7, 9, 11).

Small seismic means frequent earthquake with exceeding property of $63 \%$ within 50 years to Strong seismic means maximum considered earthquake with exceeding property of $2-3 \%$ within 50 years. 


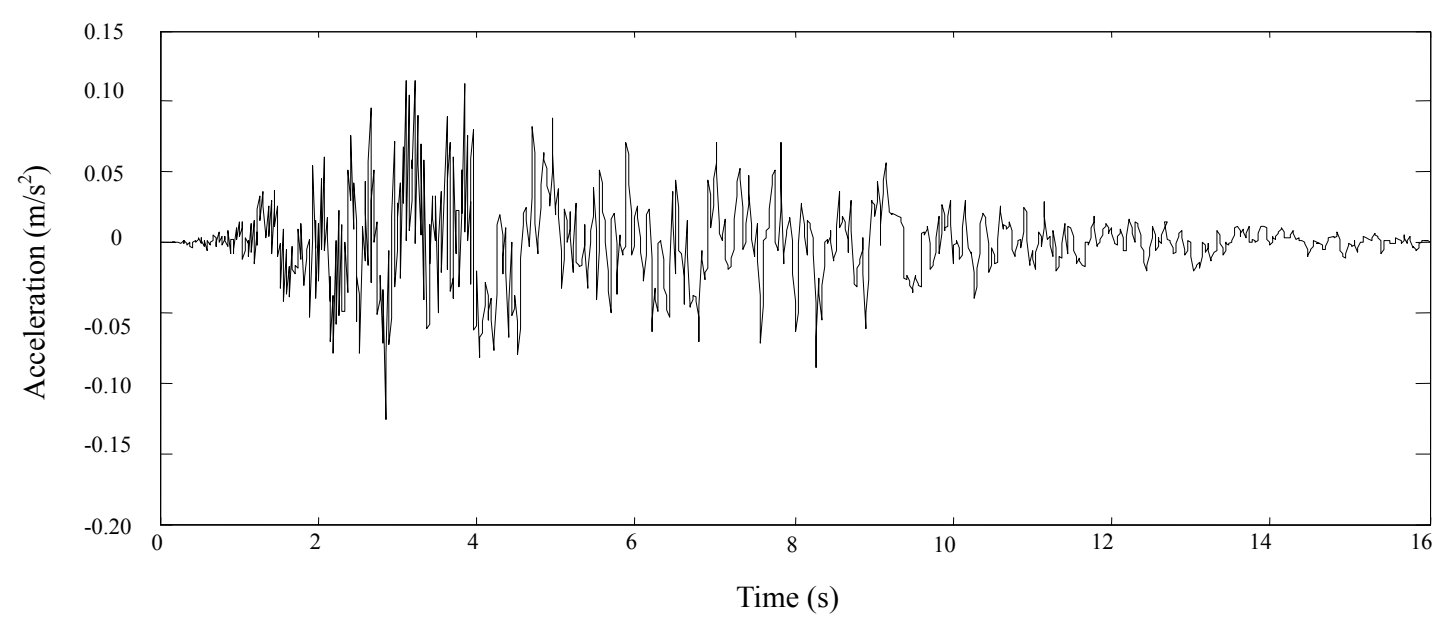

Fig. 7. type II site synthesize seismic acceleration time-history.

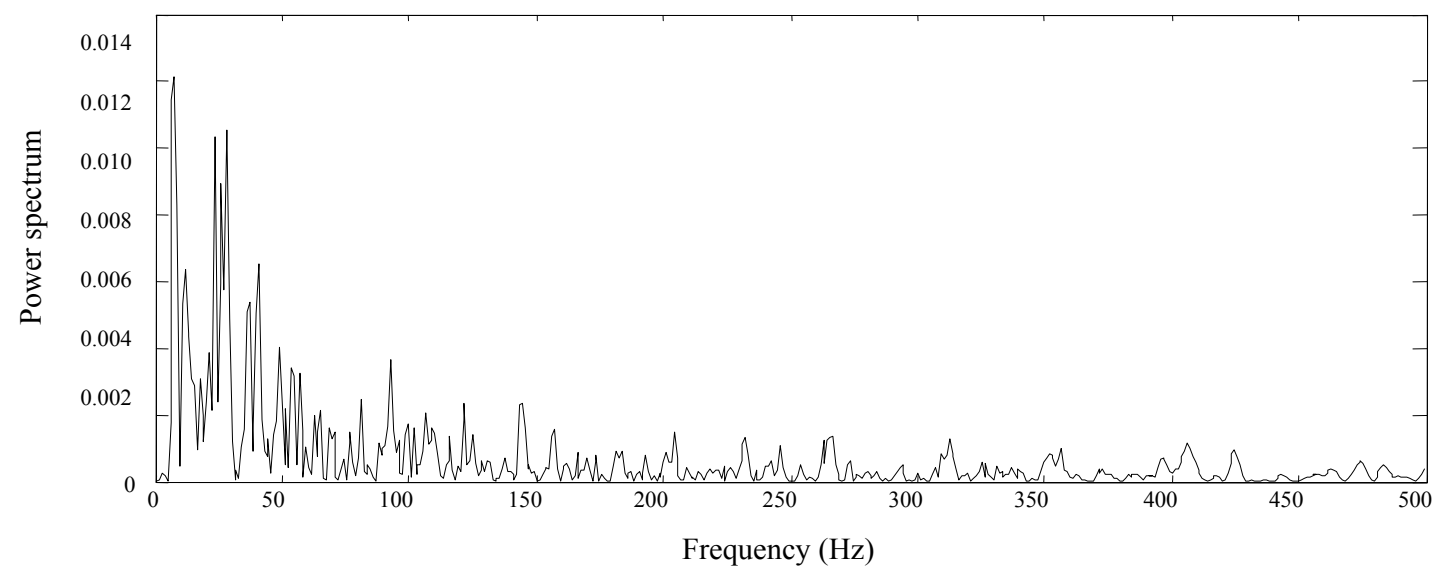

Fig. 8. type II site seismic acceleration power spectrum.

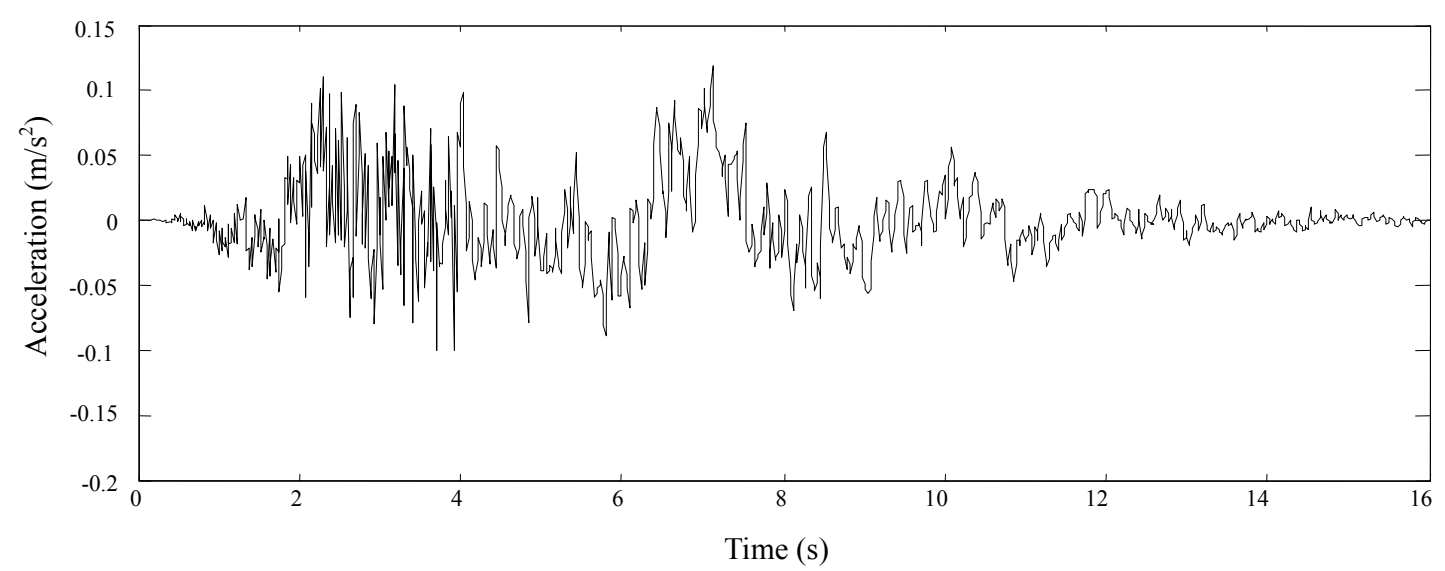

Fig. 9. Synthesized seismic acceleration time-history (site = type III). 


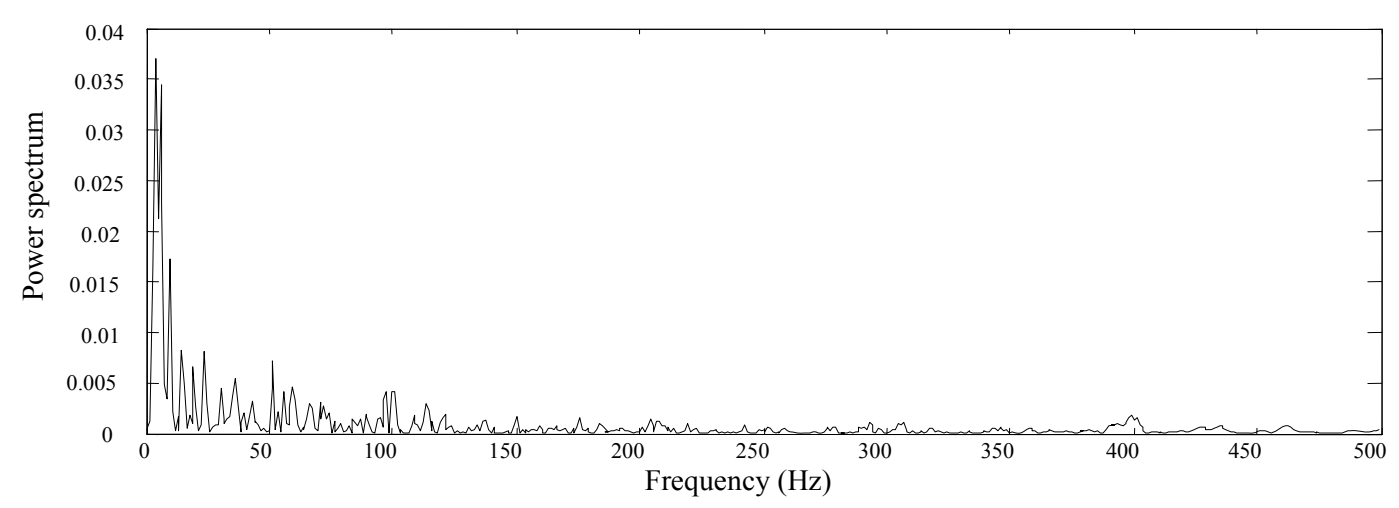

Fig. 10. Seismic acceleration power spectrum (site = type III).

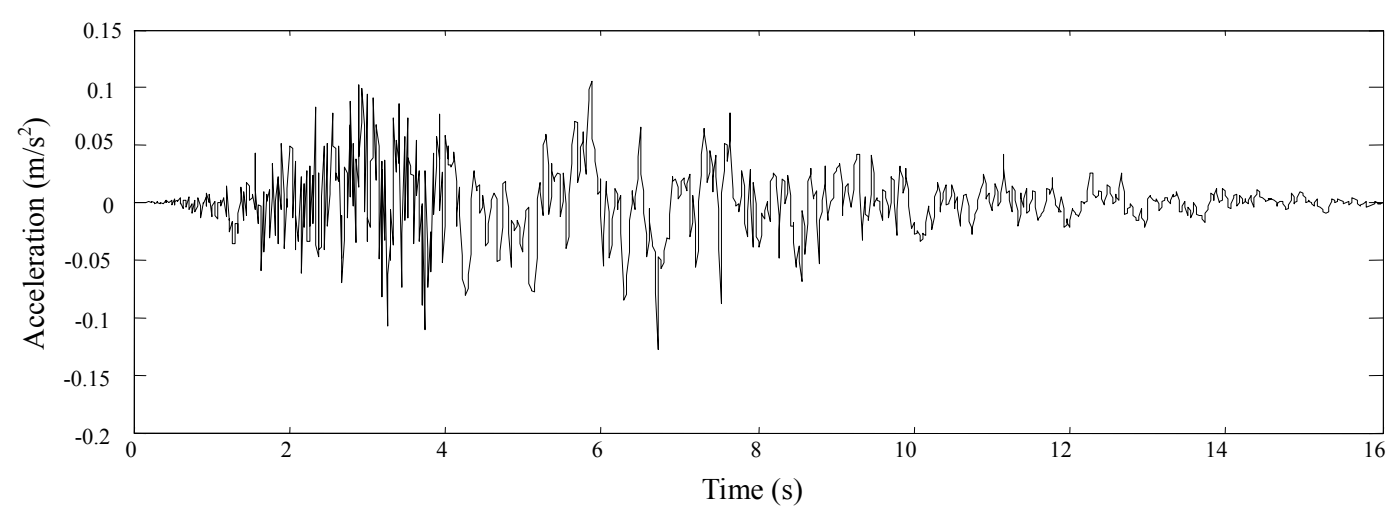

Fig. 11. Synthesized seismic acceleration time-history ( site = type IV).

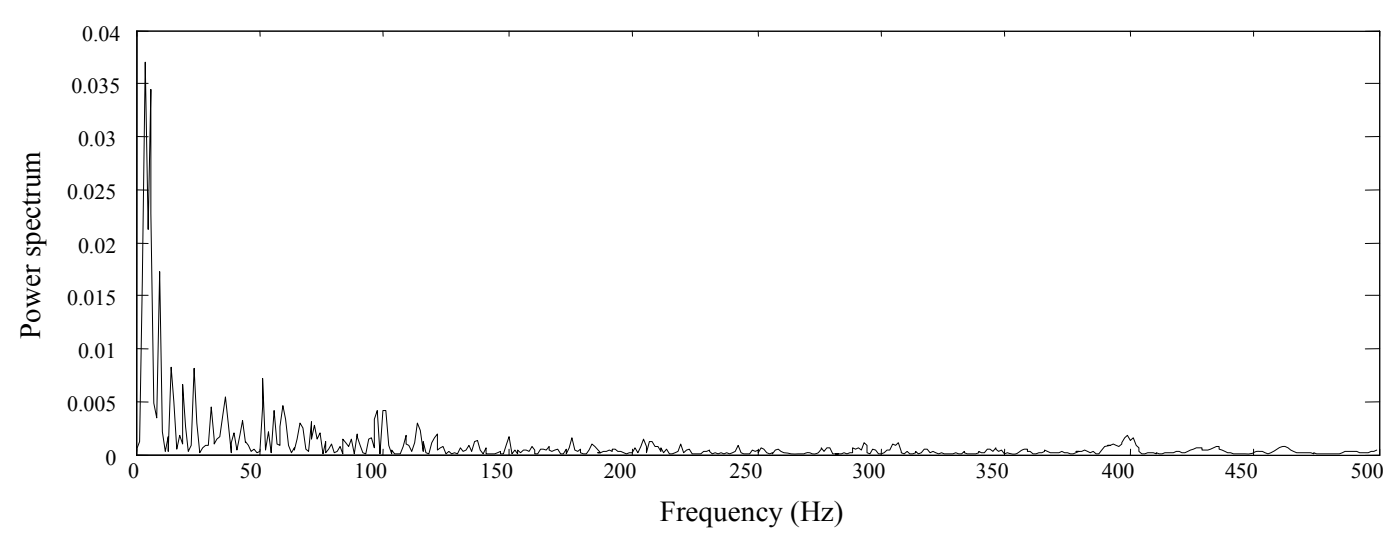

Fig. 12. Seismic acceleration power spectrum (site = type IV).

From the synthesis results above, we can obtain that seismic wave synthesized from wavelet reconstruction method contains the three main components of earthquake, and can represent the characteristics of earthquakes.

Considering the amplitude scaling coefficient $\beta$, the designed seismic wave can be rewritten as:

$$
\left\{\begin{array}{c}
\beta=\alpha_{\max } / \alpha_{\tau \max } \\
a(t)=\beta \alpha_{\tau}(t)
\end{array}\right.
$$

where $\alpha_{\max }$ is the objective peak value of acceleration in Table $4 ; \alpha_{\tau \text { max }}$ is the peak value of acceleration from 
Table 4

Maximum acceleration value used in time-history analysis $\left(\mathrm{cm} / \mathrm{s}^{2}\right)$

\begin{tabular}{lcccc}
\hline Seismic effect & 6 degree & 7 degree & 8 degree & 9 degree \\
\hline Small seismic & 18 & $35(55)$ & $70(110)$ & 140 \\
Strong seismic & - & $220(310)$ & $400(510)$ & 620 \\
\hline
\end{tabular}

Note: For cases with the design seismic accelerations of $1.5 \mathrm{~m} / \mathrm{s}^{2}$

(7 degree) or $3 \mathrm{~m} / \mathrm{s}^{2}$ (8 degree), values in the brackets are applied.

wavelet reconstruction method; $a(t)$ is the objective seismic wave; $\alpha_{\tau}(t)$ is the wavelet reconstructed acceleration function.

From Figs 7, 9, 11, the frequency corresponding to the characteristic period is calculated as $1 / 0.45=2.2$ (type II site), $1 / 0.65=1.5$ (type III site), and 1/0.9=1.1 (type IV site), respectively, which are close to those in the power spectrum curves (Figs 8, 10, 12). With variable time-frequency windows (size factor $a$ in Eqs (6) and (7)) able to widen (or narrow) automatically for high (or low) signal components, wavelet functions are theoretically nonstationary. Compared with the trigonometric series, wavelet functions have faster attenuation property in both time domain and frequency domain. Therefore synthesized ground motion samples based on the wavelet reconstruction can represent seismic characteristics better.

Although the paper focuses on Chinese standard, the wavelet reconstruction method is also applicable to other seismic design codes only if we replace the corresponding design spectrum in Eq. (20).

\section{Influencing factors}

\subsection{Number of delamination}

In Eq. (12), the wavelet frequency band $[\omega]$ is included. During the synthesis procedure, duration time, time steps and number of sampling are assumed in advance; thus, the width of frequency band is dominated by the number of wavelet delamination. According to the multi-resolution, the signal $x^{0}(t)$ can be obtained step by step:

$$
\begin{aligned}
x^{0}(t) & =\sum_{k} c_{0}^{k} \varphi_{0}^{k}(t)=\sum_{k} c_{-1}^{k} \varphi_{-1}^{k}(t)+\sum_{k} d_{-1}^{k} \psi_{-1}^{k}(t)=\ldots \\
& =\sum_{k} c_{-n}^{k} \varphi_{-n}^{k}(t)+\sum_{k} d_{-n}^{k} \psi_{-n}^{k}(t)+\ldots+\sum_{k} d_{-1}^{k} \psi_{-1}^{k}(t)
\end{aligned}
$$

Being delaminated, the signal $x^{0}(t)$ is divided into a low pass frequency band $\left[0, \frac{f_{s}}{2^{n}}\right]$ and $n$ high pass frequency band

$$
\left[\frac{f_{s}}{2^{n}}, \frac{f_{s}}{2^{(n-1)}}\right] \ldots\left[\frac{f_{s}}{2}, f_{s}\right] .
$$

When $n \rightarrow \infty,\left[0, \frac{f_{s}}{2^{n}}\right] \rightarrow 0$. Therefore, the signal of low pass can be ignored, and it can be approached as:

$$
x^{0}(t)=\sum_{k} d_{-n}^{k} \psi_{-n}^{k}(t)+\ldots+\sum_{k} d_{-1}^{k} \psi_{-1}^{k}(t)
$$

In general, the larger the number of delamination is, the less distortion the signal has. On the other hand, increasing $n$ will result in the increase of calculation exponentially. Therefore, an appropriate $n$ will meet the accuracy requirement of the synthesis and minimize the calculation at the same time. A relative perfect $n$ can be obtained as:

$$
n=j_{\max }-j_{\min }+1
$$

Where, $j_{\max }$ is the maximum resolution, $j_{\max }=\sqrt{2 f_{s}}-1 ; j_{\min }$ is the minimum resolution, and it can be determined according to the relationship between $n$ and $j_{\max }$.

In Example 1, 8 frequency bands are used to synthesize seismic wave in Fig. 13, where $\mathrm{d} 1$ is the signal of frequency band $\left[\frac{f_{s}}{2}, f_{s}\right], \mathrm{d} 2$ is the signal of frequency band $\left[\frac{f_{s}}{2^{2}}, \frac{f_{s}}{2}\right], \mathrm{d} 3$ is the signal of frequency band $\left[\frac{f_{s}}{2^{3}}, \frac{f_{s}}{2^{2}}\right]$ and so on. From Fig. 13, it can be seen obviously that most of signals pass from high pass band. In general, the larger the band number is, the less the signal passes. But for the whole synthesis signal, the larger the $n$, the less distortion the signal has. 


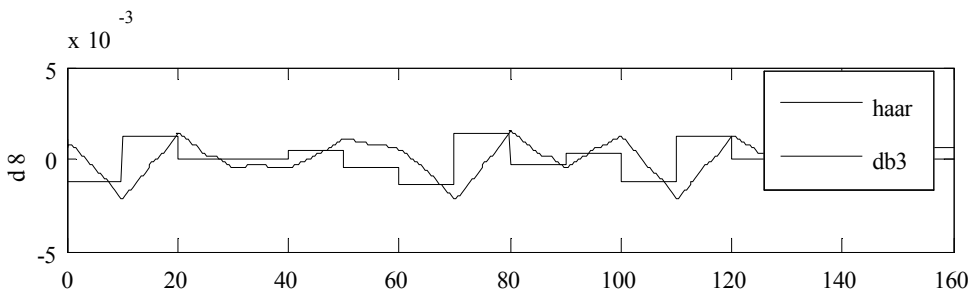

$$
\times 10^{-3}
$$
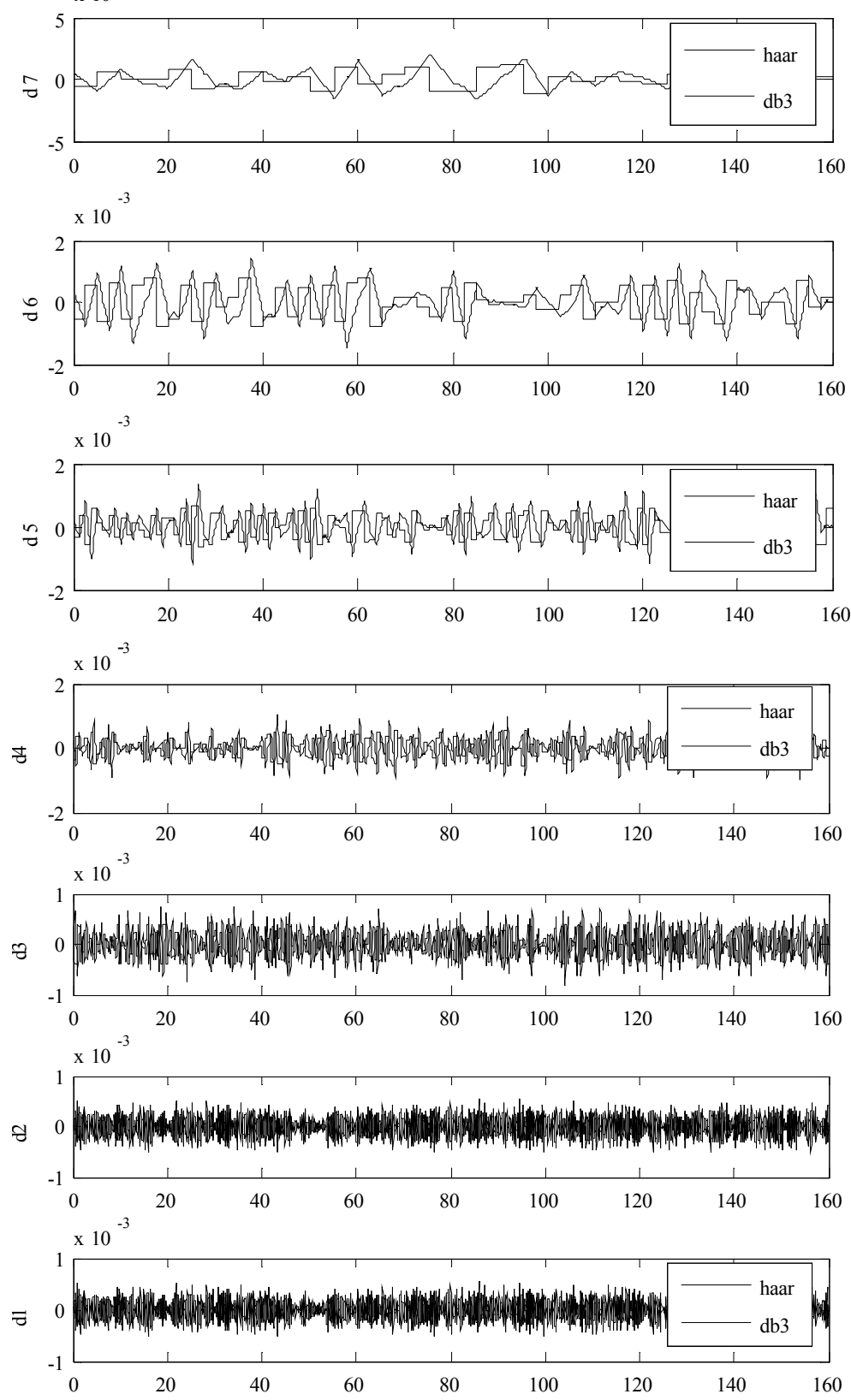

Fig. 13. Signal of different frequency band in synthesis procedure. 

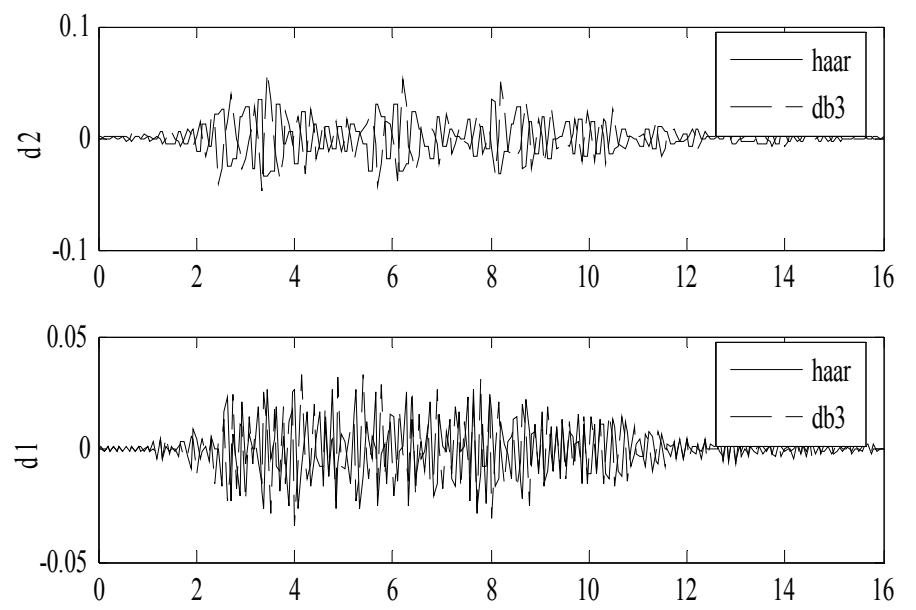

Fig. 14. Comparison of different wavelet bases in synthesis procedure.

\subsection{Wavelet function}

In wavelet reconstruction procedure, the choice of wavelet function will affect the final result. At present, there is no specific criterion in selecting wavelet functions. Generally, by comparing the errors between the signal results using wavelet method and the theoretical results, the quality of the selected wavelet bases may be determined. According to different criteria, wavelet bases have different types. The choosing criterion of wavelet probably has 4 points: (1) the support length of $\phi(t)$ and $\psi(t)$, i.e. when the time tends to infinity $\phi(t)$ and $\psi(t)$ converge to 0 from a finite value; (2) symmetry; (3) the disappear orders of $\phi(t)$ and $\psi(t)$; (4) regularity.

Generally, a symmetric wavelet has no phase aberration during transformation. A good regularity wavelet function tends to obtain smooth reconstruction curve. The numerical simulation shows that when the vibration waves are synthesized using wavelet reconstruction method, the higher the disappearing orders of basis function of wavelet is, the more smooth the curve is, and the better the effect is.

In Example 2, Daubechies function and haar function are used to synthesize seismic wave in Fig. 14, respectively. The disappearing order of db3(Daubechies function) is 3, and that of haar wavelet function is 1 . It can be seen obviously that the synthesis wave in $\mathrm{db} 3$ wavelet base has a smoother curve and is more non-stationary than that of haar. Db3 wavelet base is obviously better than haar in synthesizing seismic waves.

\section{Discussion and conclusions}

From the numerical simulation, it can be seen that waves synthesized by wavelet reconstruction are able to reflect the properties of both stationary and non-stationary vibration waves considering amplitude, duration time, frequency spectrum, stationarity in time domain and non-stationary in frequency domain and so on.

Because the simulation object of this method is realized by controlling the energy in each frequency band, and the wavelet coefficient with the same scale is approached step by step from low frequency to high frequency consequently, theoretically the simulation is accurate from low to high frequency.

There are many factors affecting the vibration synthesis, e.g. the number of delamination and the choice of wavelet function. Generally, large number of delamination can improve the quality of vibration synthesis, but will increase the amount of calculation exponentially. Thus an appropriate delamination number should be determined during the synthesis.

Currently, there is no standard in selecting wavelet functions. By comparing the errors between signal results using wavelet method and the theoretical results, the quality of the wavelet function may be determined. Generally, symmetric wavelet has no phase perturbation. The wavelet with good regularity can obtain a smooth reconstruc- 
tion curve easily. Numerical simulation shows that during the wavelet reconstruction procedure, the higher the disappearing order of wavelet function is, the smoother the curve becomes, the better the effect is.

In summary: (1) the synthesis of vibration waves based on wavelet reconstruction method contains main components of vibration, and can reflect the main properties of practical vibrations. (2) Wavelet reconstruction can simulate both stationary and non-stationary process. For the non-stationary (such as seismic) process, it has a better non-stationarity in time-frequency domain than the trigonometric series. (3) During the wavelet based synthesis procedure, it is necessary to select a wavelet function with high disappearing orders. (4) The delamination number of frequency band is important to the quality of synthesis waves. An appropriate delamination number should not only meet the accuracy requirement of synthesis waves but also simplify the calculation procedure.

\section{References}

[1] C.Q. Cai and J.W. Shen, Time-domain superposition of the artificial seismic wave and response spectrum for the whole approximating technology, Acta Seismological Sinica 19(1) (1997), 71-78.

[2] Y. Cao, H. Xiang and Y. Zhou, Simulation of stochastic wind velocity field on long-span bridges, Journal of Engineering Mechanics-ASCE 126 (1) (2000), 1-6.

[3] J.P. Conte and B.F. Peng, Fully nonstationary analytical earthquake ground-motion model, Journal of Engineering Mechanics-ASCE 23(1) (1997), 15-24.

[4] D.G. Deng and L.Z. Peng, Wavelet analysis, Mathematical Progress 20(3) (1991), 294-310.

[5] G. Deodatis and M. Shinozuka, Simulation of seismic ground motion using stochastic waves, Journal of Engineering Mechanics-ASCE 115(12) (1989), 2723-2737.

[6] L.E. Goodman, E. Rosenbueth and N.M. Newmark, Aseismic design of firmly founded elastic structures, Transaction-ASCE 120(1) (1955), $782-802$.

[7] T. Harada and M. Shinozuka, Stochastic analysis of seismic ground motions in space and time, Proceedings of the $9^{\text {th }}$ World Conference on Earthquake Engineering, Tokoyo-Japan (1988), 825-830.

[8] G W. Housner, Characteristics of strong-motion earthquakes, Bulletin of Seismological Society of America 37(1) (1947), 19-31.

[9] Y.B. Ji, S.R. Qing and B.P. Tang, Time-frequency analysis based on multi-resolution, Journal of Vibration and Shock 21(1) (2002), 12-15.

[10] A. Kareem and T. Kijewski, $7^{\text {th }}$ US national conference on wind engineering: a summary of papers, Journal of Wind Engineering and Aerodynamics 62(1) (1996), 81-129.

[11] H. Kawakami, Simulation of space-time variation of earthquake ground motion including a recorded time history, Proceedings of Japan Society of Civil Engineers 410(1-12) (1989), 435-443.

[12] J. Kiyono, K. Toki, T. Sato and H. Mizutani, Simulation of stochastic waves in a non-homogeneous random field, Soil Dynamics and Earthquake Engineering 14(5) (1995), 387-396.

[13] K.S. Kumar and T. Stathopoulos, Computer simulation of fluctuating wind pressures on low building roofs, Journal of Wind Engineering and Industrial Aerodynamics 69-71 (1997), 485-495.

[14] Y. Li and A. Kareem, Simulation of multivariate random processes: hybrid DFT and digital filter approach, Journal of Engineering Mechanics 119(5) (1991), 1028-1098.

[15] N.M. Newmark, Effects of earthquake on dams and embankments, Geotechnique 15(2) (1965), 139-159.

[16] O. Ramadan and M. Novak, Simulation of spatially incoherent random ground motions, Journal of Engineering Mechanics-ASCE 119(5) (1993), 997-1016.

[17] M. Shinozuka, Simulation of multivariate and multidimensional random processes, Journal of Acoustical Society of America 49(1) (1971), $357-368$.

[18] M. Shinozuka and C.M. Jan, Digital simulation of random processes and its applications, Journal of Sound and Vibration 25(1) (1972), $111-128$.

[19] M. Shrinkhande and V.K. Gupta, On the characterisation of the phasespectrum for strong motion synthesis, Journal of Earthquake Engineering 5 (4) (2001), 465-482.

[20] X.T. Zhang, Wind vibration analysis based on random vibration theory and evaluation for loading code at home and abroad, Proceedings of the $10^{\text {th }}$ Chinese National Wind Engineering of Structures Conference, Guilin-China (2001), 101-106.

[21] H.L. Wong and M.D. Trifunac, Generation of artificial strong motion accelerograms, Earthquake Engineering and Structural Dynamics 7 (1979), 509-527. 

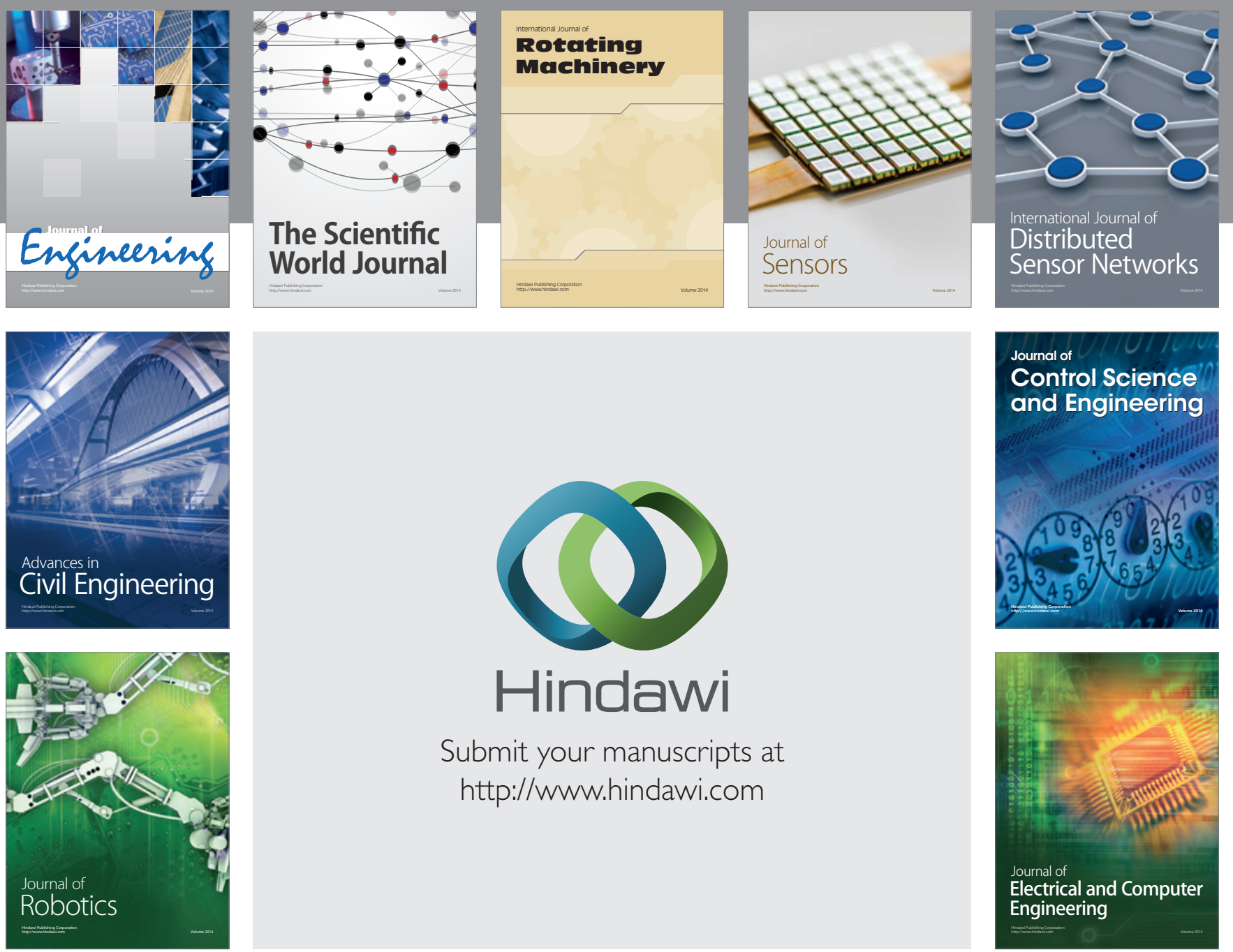

Submit your manuscripts at

http://www.hindawi.com
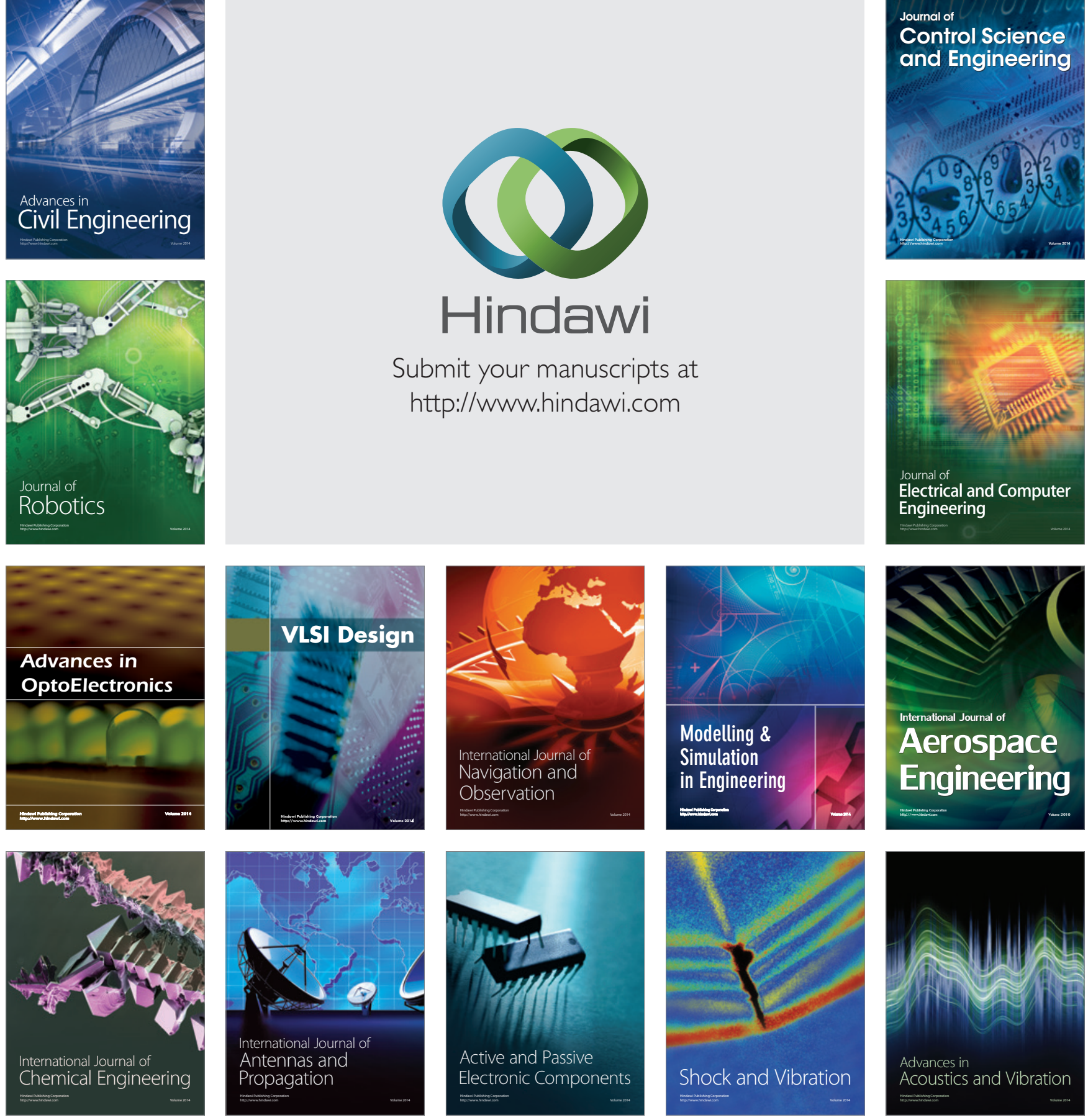